\title{
Manifold Distance-Based Over-Sampling Technique for Class Imbalance Learning
}

\author{
Lingkai Yang, Yinan Guo, Jian Cheng \\ School of Information and Control Engineering, China University of \\ Mining and Technology, Xuzhou, 221008, Jiangsu, China, +86-15150024213 \\ yanglk@cumt.edu.cn, nanfly@126.com, chengjian@cumt.edu.cn
}

\begin{abstract}
Over-sampling technology for handling the class imbalanced problem generates more minority samples to balance the dataset size of different classes. However, sampling in original data space is ineffective as the data in different classes is overlapped or disjunct. Based on this, a new minority sample is presented in terms of the manifold distance rather than Euclidean distance. The overlapped majority and minority samples apt to distribute in fully disjunct subspaces from the view of manifold learning. Moreover, it can avoid generating samples between the minority data locating far away in manifold space. Experiments on 23 UCI datasets show that the proposed method has the better classification accuracy.
\end{abstract}

\section{Introduction}

The datasets in many real classification problems are imbalanced. Dealing with class imbalanced problem normally employs data-, algorithm- or hybrid-level approaches. SMOTE(Chawla et al. 2002), as a data-level method, created new minority examples along the line between a minority class sample and its neighbors. $\operatorname{ADASYN}(\mathrm{He}$ et al. 2008) generated more data for the hard to learn minority class examples. However, over-sampling is easy to generate a wrong-labeled new sample due to the overlapped or disjunct data in different classes. Many data-level approaches employed the clustering methods to identify and preserve original data space for class imbalanced problem with small disjunct samples. MWMOTE(Barua et al. 2014) created the new samples within clusters of datapoints. ECO-Ensemble(Lim, Goh, and Tan 2017) combined oversampling strategies with the clustering methods to generate synthetic samples in each minority clustering. ACOSampling(Yu, Ni, and Zhao 2013) is an under-sampling algorithm to retain important majority samples. Without loss of generality, manifold learning extracts the essential structure of the original dataset by the manifold distance, and maps them to a low-dimensional manifold easy to be classified. Based on this, a new minority sample is generated in terms of the manifold distance rather than Euclidean distance. Especially, the overlapped minority samples may be separable in manifold space. For disjunct minority samples, a new

Copyright (C) 2019, Association for the Advancement of Artificial Intelligence (www.aaai.org). All rights reserved. sample is produced around a original minority sample in terms of the neighbors found in manifold space.

\section{The Framework of MDOTE}

The key issue of manifold distance-based over-sampling technique(MDOTE) is to generate minority samples along the line of a minority sample and its manifold distance extracted neighbors. An under-sampling strategy $(U S)$ is firstly implemented to remove redundant majority samples when all of the $k_{1}$ neighbors belong to majority class, with the purpose of building a brief balanced dataset. Based on this, a new minority sample is generated in original space based on a minority sample and one of its neighbors measured by manifold distance. LLE(Roweis and Saul 2000) is employed to extract the neighbors of minority samples. The framework of MDOTE is listed in Algorithm 1.

\footnotetext{
Algorithm 1 MDOTE

Require: Training $\operatorname{set}\left(O, O^{y}\right) ; k_{1}, k_{2}$ and $k_{3} ;$ ndim.

Ensure: The balanced dataset $X_{b}$, containing both the samples in $U$ and $X_{\text {new }}$.

1: Removing majority samples whose $k_{1}$ neighbors are all belonging to majority class,

$$
\left(U, U^{y}\right)=U S\left(O, O^{y}, k_{1}\right)
$$

2: Mapping the samples of $U$ to LLE space and extracting neighbors,

$$
\text { indices }=M N E\left(U, k_{2}, n \operatorname{dim}, k_{3}\right)
$$

3: Calculating the totally number of minority samples need to be generated,

$$
g=u_{l}-u_{s}
$$

4: Do for $i=1,2, \ldots, g$

5: Choosing one minority sample $x_{1}$ from $U$ and another minority sample $x_{2}$ from its neighbors in indices.

6: Generating new minority samples between $x_{1}$ and $x_{2}$.

$$
x_{\text {new }}=\left(x_{2}-x_{1}\right) \times \lambda+x_{1}
$$

7: End loop

Here, $U$ is the dataset after under-sampling. $M N E$ represents the neighbor extraction method. indices denotes 
Table 1: UCI Datasets

\begin{tabular}{cccc}
\hline & Dataset & & Dataset \\
\hline 1 & Abalone_18v9 & 13 & Vehicle_VANvALL \\
2 & CTG_PvN & 14 & Vehicle_SAABvALL \\
3 & CTG_SvN & 15 & Vehicle_BUSvALL \\
4 & Statland_4v12 & 16 & Wine_3vALL \\
5 & Libra_123vALL & 17 & Wine_2vALL \\
6 & Libra_789vALL & 18 & BreastCancer_MvB \\
7 & Yeast_ME1vNUC & 19 & Ionosphere_BvG \\
8 & Yeast_ME2vCYT & 20 & PageBlocks_4v2 \\
9 & Yeast_ME2vNUC & 21 & PageBlocks_5v2 \\
10 & Yeast_ME3vCYT & 22 & Segment_4v123 \\
11 & Yeast_ME3vNUC & 23 & Segment_5v123 \\
12 & Ecoli_OMvCP & \\
\hline
\end{tabular}

archive saving $k_{3}$ neighbors for each sample in $U$ used for over-sampling. $u_{l}$ and $u_{s}$ are the number of majority and minority samples after under-sampling. $k_{2}$ and ndim are the number of neighbors used in LLE and the output dimension respectively.

\section{Experiments}

All experiments are carried out on 23 UCI datasets (Table 1) and the proposed method is compared with SMOTE, ADASYN, MWMOTE, ACOSampling by AUC value. The number of neighbors and the output dimension of LLE have a direct impact on manifold learning, therefore, are optimized by a simple grid search through the cross-validation evaluation process. The statistical classification performance of different algorithms at 10 independent running times is listed in Table 2, and the best one for each dataset is labelled by bold. Here, SMO, ADA, MWM and ACO represent SMOTE, ADASYN, MWMOTE and ACOSampling method respectively. 'R+', 'R-' and 'pval' are the results of Wilcoxon paired signed-rank test between MDOTE and other methods. 'R+' means the ranking of MDOTE is better than another algorithm and 'pval' means the $p_{\text {value }}$ in hypothesis test. Lower 'pval' indicates that MDOTE has the better classification accuracy. As shown in Table 2, the proposed MDOTE outperforms other baselines for most tasks because the value of ' $\mathrm{R}+$ ' is larger than ' $\mathrm{R}$-' in all of the cases.

\section{Conclusion}

Manifold distance-based imbalance learning method is proposed to solve the class imbalanced problem with the overlapping and small disjunct data. The imbalanced dataset is transformed to a balanced one by generating minority samples around a original minority sample in terms of the neighbors found in manifold space. The experimental results on 23 UCI datasets show that the proposed method has the better classification accuracy. Combing the advanced optimizing techniques with MDOTE to improve the structure extracted by manifold learning is our future work.
Table 2: Comparison of AUC among different methods

\begin{tabular}{cccccc}
\hline & SMO & ADA & MWM & ACO & MDOTE \\
\hline 1 & 0.6590 & 0.6115 & 0.6250 & 0.6160 & $\mathbf{0 . 6 6 0 9}$ \\
2 & 0.9589 & 0.9649 & $\mathbf{0 . 9 6 5 0}$ & 0.9561 & 0.9561 \\
3 & 0.9492 & 0.9475 & 0.9313 & 0.9492 & $\mathbf{0 . 9 5 4 5}$ \\
4 & 0.9746 & $\mathbf{0 . 9 8 8 9}$ & 0.9775 & 0.9579 & 0.9846 \\
5 & 0.8758 & 0.9096 & 0.9069 & 0.9012 & $\mathbf{0 . 9 1 2 2}$ \\
6 & 0.9368 & 0.9493 & 0.9421 & 0.9306 & $\mathbf{0 . 9 5 2 4}$ \\
7 & 0.9673 & 0.9531 & 0.9714 & 0.9714 & $\mathbf{0 . 9 7 8 6}$ \\
8 & 0.9498 & 0.9498 & $\mathbf{0 . 9 7 8 2}$ & 0.9564 & 0.9616 \\
9 & 0.9141 & 0.9161 & 0.9373 & 0.9380 & $\mathbf{0 . 9 6 4 6}$ \\
10 & 0.9473 & 0.9371 & 0.9379 & $\mathbf{0 . 9 5 5 6}$ & 0.9454 \\
11 & 0.9349 & 0.9320 & 0.9190 & $\mathbf{0 . 9 3 6 4}$ & 0.9114 \\
12 & 0.9203 & 0.8870 & 0.8659 & $\mathbf{0 . 9 2 6 8}$ & 0.8993 \\
13 & 0.9437 & 0.9429 & 0.9472 & 0.9307 & $\mathbf{0 . 9 5 0 6}$ \\
14 & 0.8424 & 0.8077 & 0.8313 & $\mathbf{0 . 8 5 4 3}$ & 0.8345 \\
15 & 0.9515 & 0.9434 & 0.9410 & $\mathbf{0 . 9 5 6 4}$ & 0.9508 \\
16 & 0.9462 & 0.9538 & 0.9385 & $\mathbf{0 . 9 5 9 2}$ & 0.9462 \\
17 & 0.8898 & 0.8652 & 0.9401 & 0.8898 & $\mathbf{0 . 9 4 6 0}$ \\
18 & 0.9323 & $\mathbf{0 . 9 5 8 3}$ & 0.9011 & 0.9435 & 0.9414 \\
19 & 0.8594 & 0.8631 & $\mathbf{0 . 8 7 0 5}$ & 0.8205 & 0.8632 \\
20 & 0.9667 & 0.9667 & 0.9577 & $\mathbf{0 . 9 7 7 8}$ & 0.9667 \\
21 & $\mathbf{0 . 9 8 3 0}$ & 0.9630 & 0.9662 & 0.9729 & 0.9662 \\
22 & 0.9728 & 0.9684 & $\mathbf{0 . 9 7 3 1}$ & 0.9692 & 0.9647 \\
23 & $\mathbf{0 . 9 4 5 8}$ & 0.9365 & 0.9437 & 0.9280 & 0.9380 \\
R+ & 170.5 & 200.5 & 205.5 & 171.5 & - \\
R- & 105.5 & 75.5 & 70.5 & 104.5 & - \\
pval & 0.3051 & 0.0619 & 0.0424 & 0.3155 & - \\
\hline & & & & &
\end{tabular}

\section{Acknowledgments}

This work is jointly supported by National Natural Science Foundation of China under Grant 61573361, National Key Research and Development Program under Grant 2016 YFC0801406.

\section{References}

Barua, S.; Islam, M. M.; Yao, X.; and Murase, K. 2014. Mwmotemajority weighted minority oversampling technique for imbalanced data set learning. IEEE Transactions on Knowledge and Data Engineering 26(2):405-425.

Chawla, N. V.; Bowyer, K. W.; Hall, L. O.; and Kegelmeyer, W. P. 2002. Smote: synthetic minority over-sampling technique. Journal of Artificial Intelligence Research 16(1):321-357.

He, H.; Bai, Y.; Garcia, E. A.; and Li, S. 2008. Adasyn: Adaptive synthetic sampling approach for imbalanced learning. In IEEE International Joint Conference on Neural Networks, 1322-1328.

Lim, P.; Goh, C. K.; and Tan, K. C. 2017. Evolutionary clusterbased synthetic oversampling ensemble (eco-ensemble) for imbalance learning. IEEE transactions on cybernetics 47(9):2850-2861.

Roweis, S. T., and Saul, L. K. 2000. Nonlinear dimensionality reduction by locally linear embedding. Science 290(5500):23232326.

Yu, H.; Ni, J.; and Zhao, J. 2013. Acosampling: An ant colony optimization-based undersampling method for classifying imbalanced dna microarray data. Neurocomputing 101(2):309-318. 